\title{
Theory overview of charm physics
}

\author{
Svjetlana Fajfer ${ }^{* \dagger}$ \\ Department of Physics, University of Ljubljana, Jadranska 19 and J. Stefan Institute, Jamova 39, \\ 1000 Ljubljana, Slovenia \\ svjetlana.fajfer@ijs.si
}

Recent developments in charm physics within the standard model and beyond are reviewed. The precision study of leptonic charm meson decays accompanied with the constraints coming from $\mathrm{K}$, $\mathrm{B}$ and $\tau$ physics enables to determine constraints on models of new physics. The standard model contributions in the $D^{0}-\bar{D}^{0}$ system are still under investigation including operators of higher dimensions. New physics models such as the Littlest Higgs model or warped extra-dimensional models might modify the amount of $\mathrm{CP}$ violation, although $\mathrm{CP}$ violating asymmetries are still predicted to be at most of the order $1 \%$. The forward-backward asymmetry in the top anti-top pair production at the Tevatron is opening a new window in the study of new physics in the charm sector. A few of the models considered to explain the observed asymmetry might modify flavor changing neutral current mediated charm processes. New physics searches in rare charm decays are discussed. It is pointed out that in most models new physics effects in rare charm decays are insignificant.

The 13th International Conference on B-Physics at Hadron Machines - Beauty2011, April 04-08, 2011 Amsterdam, The Netherlands

\footnotetext{
* Speaker.

${ }^{\dagger}$ Thanks to all collaborators. Work has been supported by ARRS, Slovenia
} 
During the last few decades, flavor physics has continuously played an extremely important role in constraining models for new physics. Contrary to Flavor Changing Neutral Current (FCNC) processes of $K$ and $B$ mesons, charm FCNC physics was not considered very attractive. On the one hand, the charm quark sector provides us with an unique opportunity to test the predictions of the Standard Model (SM) and its extensions in the up quark sector. On the other hand, the FCNC processes involving charm quarks are strongly affected by the presence of non-perturbative physics, since the masses of $s$ and $d$ quarks dominating the SM loop contributions are smaller than QCD scale. Additionally, in the $\Delta C=2$ and FCNC $\Delta C=1$ transitions GIM cancellations play a very important role. The down quarks are now intermediate states and when their masses are accompanied by appropriate CKM matrix elements often strong cancellations occur. This makes precise SM predictions and searches for New Physics (NP) rather difficult. Finally, in many theoretical studies the $b$ quark can be treated as static, while the $c$ quark is not heavy enough and corrections of higher orders in $1 / m_{c}$ expansion become very important. This lecture discusses recent results on charm meson leptonic and semileptonic decays, $D^{0}-\bar{D}^{0}$ oscillations, $\mathrm{CP}$ violation in the charm system and rare charm decays. Theoretical improvements within SM are considered and confronted with recent experimental results. Concerning search for NP, correlations between $\Delta C=2$ and $\Delta C=1$ processes are discussed, but also interplay of NP in $K, B$ and charm sectors. Implications of NP searches involving charm for the recent intriguing results at the Tevatron in the top anti-top pair production are discussed.

\section{Leptonic charm decays}

Charm meson leptonic decays are very useful in providing an independent determination of the CKM matrix elements $V_{c s}$ and $V_{c d}$, but also a possibility to test theoretical tools such as Lattice QCD and investigate the validity of heavy quark and operator product expansions in addressing perturbative and power corrections. Important nonperturbative parameters entering the discussion are the decay constants $f_{D_{q}}$ defined as $<0\left|\bar{q} \gamma_{\mu} \gamma_{5} c\right| D_{q}(p)>=i f_{D_{q}} p_{\mu}$. During the past six years the experimental results from CLEO, Belle and BaBar had disagreed with Lattice QCD results (the socalled " $D_{s}$ puzzle"), stimulating many NP analyses. The results of the most recent lattice study of Fermilab Lattice and Milk Collaboration [1] are however in good agreement with the latest HFAG world average experimental values [2]

$$
\begin{aligned}
f_{D_{s}}^{\text {lat. }} & =261 \pm 8 \pm 5 \mathrm{MeV}, \quad f_{D_{s}}^{\text {exp. }}=257.3 \pm 5.3 \mathrm{MeV}, \\
\left(f_{D_{s}} / f_{D}\right)^{\text {lat. }} & =1.19 . \pm 0.01 \pm 0.02 \mathrm{MeV} .
\end{aligned}
$$

Although the disagreement between lattice and experimental results has almost disappeared, leptonic charm decays still offer many useful constraints on NP. It was pointed out in [3] that charged Higgs or leptoquarks can contribute at the tree level. In scenarios with a charged Higgs interfering with the SM, useful bounds on $\tan \beta$ vs. the mass of the charged Higgs can be obtained, as presented in $[4,5]$. The presence of weak triplet, doublet or singlet leptoquarks in charm meson leptonic decays was investigated in [6]. The triplet leptoquarks with electric charge $-1 / 3$ can contribute to $D_{q} \rightarrow \ell v_{\ell}$ but also to $\tau \rightarrow \eta \mu, \tau \rightarrow \pi \mu, K \rightarrow \mu v_{\mu}, K^{+} \rightarrow \pi^{+} v \bar{v}, K_{L} \rightarrow \mu^{+} \mu^{-}$and to the ratio $B R(\tau \rightarrow \pi v) / B R(\pi \rightarrow \mu v)$. Altogether, these observables exclude the triplet leptoquarks 
from affecting significantly the $D_{q} \rightarrow l v_{l}$ mode. The doublet leptoquark on the other hand, can mediate $K_{L} \rightarrow \mu^{+} \mu^{-}$and $\tau \rightarrow \eta \mu$. Then, the recent Belle upper bound on the branching ratio of $D^{0} \rightarrow \mu^{+} \mu^{-}$excludes also the doublet leptoquarks from affecting $D_{s} \rightarrow \mu v$, while the tau mode is still unconstrained. The Lagrangian describing the singlet leptoquark includes two terms. One of them can be matched to the R-parity violating minimal supersymmetric SM, where the interaction term of a right-handed down squark to quark and lepton doublets is present. However, both terms in the Lagrangian are necessary to to avoid bounds coming from $B R(K \rightarrow \mu v) / B R(\tau \rightarrow K v)$. As in the doublet case, the lack of experimental information on up-quark FCNCs involving tau leptons then leaves open the verdict on the singlet leptoquark contribution to the $D_{s} \rightarrow \tau \nu$ decay width.

\section{Semileptonic charm decays}

Inclusive charm meson decays can be used as a laboratory to test the operator product expansion techniques used in the extraction of $\left|V_{u b}\right|$ from inclusive semileptonic B decays. In this approach, a handle on the so-called weak annihilation contributions is very important to control theoretical uncertainties. Recent analyses of perturbative $\alpha_{s}$ and $1 / m_{c}$ power corrections in charm meson inclusive semileptonic decays show that there is no significant indication of weak annihilation contributions $[7,8]$.

Regarding the exclusive semileptonic modes, a recent lattice study of the semileptonic form factors in $D \rightarrow K \ell v_{\ell}$ [9] finds for the vector form factor at zero lepton momentum squared $f_{+}(0)=$ $0.747 \pm 0.011 \pm 0.015$. Comparing this value to the recent experimental results, the relevant CKM matrix element is found to be $\left|V_{c s}\right|=0.961 \pm 0.011 \pm 0.024$.

\section{3. $D^{0}-\bar{D}^{0}$ oscillations}

The $D^{0}-\bar{D}^{0}$ system is the only neutral meson system of valence up-type quarks. Within the SM, the mixing of the two flavor eigenstates is driven by intermediate states of down-type quarks. Among them, the $\mathrm{b}$ quark contributions are negligible due to the smallness of the relevant CKM matrix elements. The masses of $s$ and $d$ quarks are however, smaller then the nonperturbative QCD scale $\Lambda_{Q C D}$ implying that long distance contributions can dominate the dynamics of the $D^{0}-\bar{D}^{0}$ system. A recent fit by HFAG [2] for the four experimentally relevant observables yields

$$
\begin{aligned}
x_{D} & =\frac{\Delta M_{D}}{\Gamma_{D}}=\left(0.63_{-0.20}^{+0.19}\right) \%, y_{D}=\frac{\Delta \Gamma_{D}}{\Gamma_{D}}=(0.75 \pm 0.12) \% \\
\left|\frac{q}{p}\right| & =0.91_{-0.16}^{+0.18}, \quad \Phi=\left(-10.2_{-8.9}^{+9.4}\right)^{o} .
\end{aligned}
$$

The phase is defined as $\Phi=\operatorname{Arg}(q / p) . \Delta M$ and $\Delta \Gamma$ do not give any information on CP violation, while $|q / p| \neq 1$ and $\Phi \neq\{0, \pm \pi\}$ would signal the presence of CP violation in the charm meson system. However, this can be completely due to SM dynamics. There are presently two approaches to estimating the SM contributions: inclusive approach, based on the assumption of quark-hadron duality $[10,11,12]$, and the exclusive approach, based on the study of individual decay channels contributing to the mixing of $D$ mesons $[13,14,15]$. Both approaches suffer from large theoretical uncertainties. 
The authors of [16] recently investigated the SM CP violating effects in $D$-mixing within the inclusive approach, including perturbative $\alpha_{s}$ as well as $1 / m_{c}$ power corrections to the absorptive part of the mixing amplitude of neutral $D$ mesons. In $D^{0}-\bar{D}^{0}$ system $1 / m_{c}$ corrections are suppressed by fourth power of $m_{s}$ in $x_{D}$ and by the sixth power in $y_{D}$. It was shown that dimensions 6 and 7 operators lead to 10000 smaller value of $\Gamma_{12}$ than the current experimental value. Due to the strong GIM cancellation it might happen that the operators with the dimension 9 or 12 give dominant contributions. The authors suggest that "CP violating effects in charm meson system of the order of $10^{-3}$ to $10^{-2}$ are an unambiguous sign of new physics" [16] .

In the exclusive approach the contribution of the sum of intermediate hadronic states is estimated. Recenly [15] the sum of all two body intermediate states was reinvestigated, based on the fact that $63 \%$ of hadronic $D^{0}$ decays are two-body decays. The remaining multi-body hadronic decays contribute much less, particularly when cancellations among them are included into consideration. The contributions considered in [15] are found to be primary from the P P and V P modes leading to the estimates $x_{D} \sim 10^{-3}$ and $y_{D} \sim 10^{-3}$. As pointed out already in [17] $x_{D} \sim 1 \%$ and $y_{D} \sim 1 \%$ are possible within the SM. Due to the large uncertainty one cannot subtract these values from the experimental results and claim that the difference is due to new physics. Usually, the experimental values of $x_{D}$ and $y_{D}$ themselves are used as constraints on possible NP contributions, requiring that these do not exceed the experimental errors.

If the masses of intermediate NP particles are above the electroweak scale one can integrate out these heavy degrees of freedom and consider effective $\Delta C=2$ Lagrangian. At the scale of the charm quark mass these contributions are modified by the strong interaction effects. The running QCD effects has been known for some time [18, 19, 20]. Taking operator mixing into account, the real and imaginary parts of the corresponding Wilson coefficients at the electroweak scale have been recently constrained [21, 22, 23].

Among the possible new flavor structures beyond the SM, Minimal Flavor Violation (MFV) offers an interesting possibility that the NP shares the structure of the SM Yukawa terms. Promoting the Yukawa matrices to spurionic fields, transforming as $Y_{u}(3, \overline{3}, 1)$ and $Y_{d}(3,1, \overline{3})$ under the SM flavor group $G=S U(3)_{Q} \times S U(3)_{U} \times S U(3)_{D}$, the NP Wilson coefficients entering the effective Lagrangian, have to involve powers of $Y_{d} Y_{d}^{\dagger}$ and $Y_{u} Y_{u}^{\dagger}$. Within a non-linear realization of MFV the authors [25] found that the phase $\Phi$ in the $D^{0}-\bar{D}^{0}$ system can be related to the CP-violating phase $\gamma$ entering B physics observables.

Lately a number of models of NP beyond MFV framework have been tested in the $D^{0}-\bar{D}^{0}$ system. In models where new flavor violating effects have been moved to the up sector, the leading constraints come from charm physics. The fourth generation as the simplest SM extension in the flavor sector, has attracted a lot of attention (c.f. [26]). In [26] the strongest bounds on $\left|V_{u b^{\prime}} V_{c b^{\prime}}\right|$ were derived from the $D$-mixing analysis for the mass of $b^{\prime}$ ranging from $200-500 \mathrm{GeV}$.

\section{Implications for the top quark sector}

The measured forward-backward asymmetry in the $t \bar{t}$ production and especially at high invariant masses of the $t \bar{t}$ system at the Tevatron offer new opportunities for new physics searches at low energies. It is very intriguing that only the measured asymmetries deviate from SM predictions, while the measured production cross section and invariant mass spectrum are in reasonably good 
agreement with SM predictions. Among many models (see e.g. [27]), the exchange of a colored weak singlet scalar in the u-channel and its interference with the SM contribution can accommodate all relevant observables measured at the Tevatron. Such state appears in some of the grand unified theories and its interactions with the up-quarks are purely antisymmetric in flavor space. The resulting impact on charm and top quark physics has been systematically investigated in [28]. It turns out that one of the most important constraints on the relevant couplings comes from the experimentally measured observables related to $D^{0}-\bar{D}^{0}$ oscillations While the $t \bar{t}$ phenomenology requires the $u-t$ coupling with the exotic scalar state of the order 1, the corresponding $c-t$ coupling entering the box mediated $c \bar{u} \leftrightarrow \bar{u} c$, is then constrained by $D$-mixing to be two orders of magnitude smaller. In a class of grand unified models it has been demonstrated that these constraints affect the up-quark Yukawa couplings leading to a lopsided up-quark mass matrix [29] . Models with a color sextet weak singlet scalar can also explain observed asymmetries at the Tevatron without significant modifications of the cross section for the $t \bar{t}$ production (see e.g. [27]). However, in $D^{0}-\bar{D}^{0}$ oscillations the color sextet can contribute already at the tree level [30], resulting in even more severe constraints on the $u-c$ sextet coupling. Likewise, a new flavor changing $Z^{\prime}$ gauge boson is also successful in accomodating the Tevatron $t \bar{t}$ observables. However, the $Z^{\prime}$ mediation of the $D^{0}-\bar{D}^{0}$ oscillations at the tree level again requires the $c u Z^{\prime}$ coupling to be orders of magnitude smaller than the $t u Z^{\prime}$. On the theoretical side such alignment imposes questions about the underlying flavor structure of such models.

\section{New CP violation in charm}

The Littlest Higgs model with T-parity (LHT) offers an appealing solution to the Higgs hierarchy problem without causing problems with the electroweak constraints. It introduces a discrete symmetry called T-parity, under which the new particles can be odd so that they contribute to processes of SM particles only at the loop level. The model contains three new families of mirror quarks and consequently two additional $\mathrm{CP}$ violating phases. Parameters describing these new degrees of freedom can then be related to $\mathrm{CP}$ asymmetries in $K, B$ and $D$ decays of [31] found that LHT model can produce observable CP violating effects in the charm system. In the case of nonleptonic neutral charm meson decay to $K_{S} \Phi$ two CP violating quantities $S_{f}$ and $a_{S L}\left(D^{0}\right)$ offer tests of LHT dynamics [32]. It is interesting that indirect CP violation in the nonleptonic decays $D^{0} \rightarrow \pi^{+} \pi^{-}, D^{0} \rightarrow K^{+} K^{-}$and $D^{0} \rightarrow \Phi K_{S}$ will create the same asymmetries. If experiment will find different $\mathrm{CP}$ asymmetries, then they have to be the result of direct $\mathrm{CP}$ violation. The authors of [32] noticed that an enhancement factor of two in $a_{C P}^{d i r}$ is possible for the unconstrained set of parameters.

On the other hand, in the Randall - Sundrum model studied in [33] the CP violating parameters can reach $S_{\Phi K_{S}} \in[0.25,0.15]$ and $a_{S L}\left(D^{0}\right) \in[-0.75,0.4]$ for the set of model parameters constrained by $\left|\varepsilon_{K}\right|, Z^{0} \rightarrow \bar{b} b$, and $B_{d}-\bar{B}_{d}$ oscillations and assuming $\Gamma_{12}^{D}=-0.02$ and $0.02 \mathrm{ps}^{-1}$.

\section{Rare charm decays}

In the SM the contribution coming from penguin diagrams in $\mathrm{c} \rightarrow \mathrm{u} \gamma$ transition is strongly GIM suppressed giving a branching ratio of order $10^{-18}$ [34], while the QCD corrected effective 
Lagrangian gives $\mathrm{BR}(\mathrm{c} \rightarrow \mathrm{u} \gamma) \simeq 3 \times 10^{-8}$ [36]. A variety of models beyond the SM have been investigated and it was found that the gluino exchange diagrams [37] within the general minimal supersymmetric SM (MSSM) might lead to significant enhancements. With the updated constraints it was found that [39] $\Gamma(\mathrm{c} \rightarrow \mathrm{u} \gamma) / \Gamma_{\mathrm{D}^{0}} \leq 8 \times 10^{-7}$.

Likewise, within the SM the $c \rightarrow u l^{+} l^{-}$amplitude is given by the $\gamma$ and $Z$ penguins and $W$ box diagrams at one-loop electroweak level. It is dominated by the light quark contributions in the loops. The leading order rate for the inclusive $c \rightarrow u l^{+} l^{-}$calculated within SM [?] was found to be suppressed by QCD corrections [35]. Inclusion of renormalization group equations for Wilson coefficients gave an additional significant suppression [40] leading to the rates $\Gamma(c \rightarrow$ $\left.u e^{+} e^{-}\right) / \Gamma_{D^{0}}=2.4 \times 10^{-10}$ and $\Gamma\left(c \rightarrow u \mu^{+} \mu^{-}\right) / \Gamma_{D^{0}}=0.5 \times 10^{-10}$. These transitions are largely driven by virtual photon contributions at low dilepton mass. The leading contribution to $c \rightarrow u l^{+} l^{-}$ in the MSSM with the conserved R parity comes from one-loop diagrams [35, 37] with gluino and squarks in the loop. It proceeds via virtual photon and significantly enhances the $c \rightarrow u l^{+} l^{-}$ spectrum at small dilepton mass $m_{l l}$. The effects of the extra heavy up vector-like quark models on the decay spectrum of $D^{+} \rightarrow \pi^{+} l^{+} l^{-}$and $D_{s}^{+} \rightarrow K^{+} l^{+} l^{-}$decays were also considered [39]. It was found that there is a tiny increase of the differential decay rate in the region of large dilepton mass.

Recently $D^{0} \rightarrow \gamma \gamma$ and $D^{0} \rightarrow l^{+} l^{-}$were reconsidered in [41]. The result for the short and long distance contributions are $B R_{S D}^{2-\text { loops }}\left(D^{0} \rightarrow \gamma \gamma\right) \simeq(3.6-8.1) \times 10^{-12}, B R_{S D}^{2-\text { loops }}\left(D^{0} \rightarrow \gamma \gamma\right) \simeq$ $(3.6-8.1) \times 10^{-12}$. Current bound is quite weak with $B R_{\exp }\left(D^{0} \rightarrow \gamma \gamma\right) \sim 2.7 \times 10^{-5}$ and it seems that real improvements can be achieved only at the Super Flavor or the Super Tau-Charm factory. Short distance contributions in $D^{0} \rightarrow l^{+} l^{-}$decay lead to a very suppressed branching ratio in the SM. Therefore it is natural to consider it is as an ideal testing ground for NP effects. Reference [41] considered contributions coming from $\gamma \gamma$ intermediate states due to long distance dynamics in $D^{0} \rightarrow \gamma \gamma$ arriving at the value $B R\left(D^{0} \rightarrow \mu^{+} \mu^{-}\right) \sim(2.7-8) \times 10^{-13}$. According to their calculations, the LHT model can enhance the branching ratio for a factor of 2 .

In $[18,17]$ an operator product expansion has been used to obtain the effective Hamiltonian and explore the correlations of the NP in $D^{0}-\bar{D}^{0}$ oscillations and $D^{0} \rightarrow \mu^{+} \mu^{-}$. Although NP contributions are dominant in this decay channel they in the best case (vector-like quark models) $[18,17]$ increase the rate by a factor of 100 . The experimental bounds on $D^{0} \rightarrow \ell^{+} \ell^{-}$have recently been improved by the Belle collaboration to $B R\left(D^{0} \rightarrow \mu^{+} \mu^{-}\right) \leq 1.4 \times 10^{-7}$ and $B R\left(D^{0} \rightarrow e^{+} e^{-}\right) \leq$ $7.9 \times 10^{-8}[42]$.

\section{Conclusions}

The accumulated data on charm physics give new insights into SM contributions and further improvements of the theoretical tools are still possible. In particular the complete knowledge of the long distance contributions in the $x_{D}$ and $y_{D}$ observables in $D^{0}-\bar{D}^{0}$ oscillation is still lacking. Therefore, NP constraints could still be improved. The search for CP violating signals has progressed and a lot of models of new physics have predicted increase of $\mathrm{CP}$ violating asymmetries relative to SM results. However, they all of the order $1 \%$. The rare decay modes can be enhanced by at most two orders of magnitude in most SM extensions, still far bellow the current experimental precision. The anomalous behavior of the $t \bar{t}$ production at Tevatron opened new paths in the study of NP effects in the charm sector. If the measured asymmetries remain, this will require new 
insight into NP in charm as well. With current searches at $\mathrm{LHCb}$ and with future planned facilities including the charm physics program, we expect a stimulating and fruitful era for charm.

\section{References}

[1] Fermilab Lattice and MILC Collaboration (J. Simone (Fermilab) et al.), PoS LATTICE2010, 317 (2010).

[2] Heavy Flavor Averaging Group (HFAG), http://www.slac.stanford.edu/xorg/hfag/charm/.

[3] B. A. Dobrescu and A. S. Kronfeld, Phys. Rev. Lett. 100, 241802 (2008) [arXiv:0803.0512 [hep-ph]]

[4] A.G. Akeroyd and F. Mahmoudi, JHEP 0904121 (2009) [ arXiv:0902.2393 [hep-ph]].

[5] S. Descotes-Genon, talk given at The 4th International Workshop on Charm Physics, Beijing Oct. 21-24, 2010.

[6] I. Doršner, S. Fajfer, N. Košnik, J. Kamenik, Phys. Lett. B 682, 671 (2009) [arXiv:0906.5585 [hep-ph]].

[7] J. F. Kamenik and P. Gambino, Nucl. Phys. B 840, 424 (2010) 424 [arXiv:1004.0114 [hep-ph]].

[8] Z. Ligeti, M. Luke, A. V. Manohar, Phys. Rev. D 82 (2010) 033003 [arXiv:1003.1351 [hep-ph]].

[9] H. Na, C.T.H. Davies, E. Follana, P. Lepage, J. Shigemitsu., PoS LATTICE2010 315 (2010) [arXiv:1103.5419 [hep-lat]].

[10] H. Georgi, Phys. Lett. B 297353 (1992) .

[11] T. Ohl, G. Ricciardi, E. H. Simmons, Nucl. Phys. B 403, 605 (1993) [arXiv:hep-ph/9301212].

[12] I. Bigi and N. Uraltsev, Nucl. Phys. B 592, 92 (2001).

[13] J. Donoghue, E. Golowich, B. Holstein and J. Trampetic, Phys. Rev. D 33, 179 (1986); L. Wolfenstein, Phys. Lett. B164, 170 (1985); P. Colangelo, G. Nardulli and N. Paver, Phys. Lett. B242, 71 (1990); T.A. Kaeding, Phys. Lett. B357, 151 (1995); E. Golowich and A. A. Petrov, Phys. Lett. B 427, 172 (1998); A. A. Anselm and Y. I. Azimov, Phys. Lett. B 85, 72 (1979).

[14] A. F. Falk, Y. Grossman, Z. Ligeti and A. A. Petrov, Phys. Rev. D 65, 054034 (2002); A. F. Falk, et. al, Phys. Rev. D 69, 114021 (2004).

[15] H.-Y. Cheng, C.W. Chiang, Phys. Rev. D 81, 114020 (2010) [arXiv:1005.1106 [hep-ph]]

[16] M. Bobrowski, A. Lenz, J. Riedl, J. Rohrwild. JHEP 1003, 009 (2010) 009 [arXiv:1002.4794 [hep-ph]].

[17] A. Petrov, PoS BEAUTY2009 024 (2009) [arXiv:1003.0906 [hep-ph]].

[18] E. Golowich, S. Pakvasa and A. A. Petrov, Phys. Rev. Lett. 98, 181801 (2007).

[19] A. A. Petrov and G. K. Yeghiyan, Phys. Rev. D 77, 034018 (2008).

[20] E. Golowich, J. Hewett, S. Pakvasa and A. A. Petrov, Phys. Rev. D 76, 095009 (2007).

[21] O. Gedalia, Y. Grossman, Y. Nir and G. Perez, Phys. Rev. D 80, 055024 (2009) [arXiv:0906.1879].

[22] W. Altmannshofer, A. J. Buras and P. Paradisi, Phys. Lett. B 688, 202 (2010) arXiv:1001.3835 [hep-ph].

[23] G. Isidori, Y. Nir, G. Perez. [arXiv:1002.0900 [hep-ph]]. 
[24] D’Ambrosio, G. et al. Nucl.Phys. B 645, 155 (2002) [hep-ph/0207036].

[25] A. L. Kagan, G. Perez, T. Volansky, J. Zupan, Phys. Rev. D80, 076002 (2009) [arXiv:0903.1794 [hep-ph]].

[26] O. Eberhardt, A. Lenz, J. Rohrwild, Phys. Rev. D 82095006 (2010) [arXiv:1005.3505 [hep-ph]].

[27] M. I. Gresham, I-W. Kim, K. M. Zurek, [ arXiv:1103.3501 [hep-ph]].

[28] I. Doršner, S. Fajfer, N. Košnik, J. Kamenik, Phys. Rev. D 81, 055009 (2010) , arXiv:0912.0972 [hep-ph]

[29] I. Doršner, S. Fajfer, N. Košnik, J. Kamenik, Phys. Rev. D 82, 094015 (2010) [arXiv:1007.2604 [hep-ph]].

[30] C.-H. Chen, Phys. Lett. B 680, 133 (2009) [arXiv:0902.2620 [hep-ph]].

[31] I. Bigi, M. Blanke, A. J. Buras, S. Recksiegel, JHEP 0907, 097 (2009) [arXiv:0904.1545 [hep-ph]].

[32] I. Bigi, A. Paul, S. Recksiegel, [arXiv:1103.5785 [hep-ph]].

[33] M. Bauer, S. Casagrande, L. Grunder, U. Haisch, M. Neubert, JHEP 009, 017 (2010) [arXiv:0912.1625 [hep-ph]].

[34] G. Burdman, E. Golowich, J. Hewett and S. Pakvasa, Phys. Rev. D 52, 6383 (1995).

[35] G. Burdman, E. Golowich, J. Hewett and S. Pakvasa, Phys. Rev. D 66, 014009 (2002).

[36] C. Greub, T. Hurth, M. Misiak and D. Wyler, Phys. Lett. B 382, 415 (1996); Q. Ho Kim and X.Y. Pham, Phys. Rev. D 61, 013008 (2000).

[37] S. Prelovsek and D. Wyler, Phys. Lett. B500, 304 (2001).

[38] S. Fajfer and Sasa Prelovsek, Phys. Rev. D 73, 054026 (2006).

[39] S. Fajfer, N. Košnik and S. Prelovsek, Phys. Rev. D 76074010 (2007) [arXiv:0706.1133 [hep-ph]].

[40] S. Fajfer, P. Singer, J. Zupan, Eur. Phys.J. C 27, 201 (2003).

[41] I. Bigi, A. Paul, S. Recksiegel, [arXiv:1008.3141 [hep-ph]].

[42] M, Starič (Belle collaboration) PoS ICHEP2010 (2010) 266. 